\title{
ON THE INFLUENCE
}

\section{OP \\ INADEQUATE OPERATIONS ON THE THEORY OF CANCER.}

\author{
BY \\ CHARLES H. MOORE, F.R.C.S., \\ VICE-PRESIDENT OF THE SOCIETY, SURGEON TO THE MIDDLESEX, \\ AND TO ST. LUKE's, HOSPITALS.
}

Received April 7th.-Read May 28th, 1867.

Theories respecting the nature of Cancer involve the consideration of many facts. An explanation of the disease, to be correct, must reconcile its general relations, such as peculiarities in the family or race, personal habits, and external or geographical condition of the patient, with the various phenomena presented by the disease itself; and with the resemblances and distinctions which exist between it and other tumours. So multitudinous and apparently diverse are the facts on which the rival theories depend that the subject is still shrouded in much obscurity. But much also of the confusion relating to it arises from error and indefiniteness in the observation of the facts themselves.

I do not propose to discuss these general questions in the present paper, but to contribute one of the many series of facts which must concur to their solution. I desire to ascertain if there be a traceable method in the recurrence of 
Cancer after operations, or if the renewed disease affect the part confusedly; to discover if Cancer be orderly in its return, conforming to the rule of local functions, or if its morbid material be shed forth into an organ, or a region, or into the body as a whole, under an influence which is superior to local conditions.

The bearing of such an inquiry upon the chief theories of Cancer appears to be this :-

There is a theory which includes in the causation of the disease a constitutional malady, something apart from the palpable local tumours, which hovers (the phrase is Broca's) over the system, ready to alight on any or on many different parts of the body. If the recurrence of Cancer be wholly explicable by conditions in the part which has been the seat of an operation, the addition of another cause is superfluous.

There is again a theory which attributes Cancer to what may be called a textural or an organic diathesis. It is illustrated by the phenomena of many innocent tumours; as when multitudes of villous tumours are strown along a mucous membrane, or neuromas are strung on all the nerves. And it differs from the former theory at least in this, that it restricts the cause of the disease within the organ or texture affected by it. But if it thus limit and localize the cause, this theory likewise connects it with the textural or organic functions, and requires that the tendency to disease should be co-extensive with the respective organ or texture. In the case of double organs therefore, on the removal of one that is cancerous, a prominent liability to the same disease should be transferred to its fellow. I am not prepared to deny that perverted function influences the first outbreak of some Cancers, for there do indistinctly appear to be various causes concerned in setting up similar Cancers, of which causes that may be one. But the theory as a whole may be so far tested by recurrence after operations as to show whether the continued disease belong or not to the organ.

Again, Cancer is sometimes regarded as regional. As socalled supplemental organs are formed in the vicinity of a 
principal organic mass, or as some tumours bear a resemblance to the structure near which they originate, so Cancer is held to arise within a region by successive independent growths beyond the limits of the first tumour and of the organ in which it sprang. This theory too may to some extent be tested by the method in which Cancer returns after operations; but when recurrence is late, and not precisely local, the distinction between regional Cancer and a yet more strictly local growth is not easy to draw. It is then necessary to discern between a recurrence traceable to incompleteness of the operation and that return of the disease in which a morbid tendency may be supposed to possess every several structure throughout a region, independently of the extirpated portion which first formed a tumour. No help in solving the question is to be procured by apportioning the irritation of the disease to the blastema or to the cell. It can only be decided by accurate and repeated observations of the mode of recurrence. And there are two elements of uncertainty in the question; uncertainty, namely, whether the operation comprehended all the area over which material from the primary tumour was diffused, and uncertainty respecting the possible duration of inactive life in a fragment of Cancer, severed in the operation from the primary tumour and left in the tissues. When Cancer returns after operations, is it like caries, to which the healthy teeth continue independently liable, though the first diseased tooth have been drawn, or does it correspond to a carious stump, from which only a part of the disease has been taken by breaking off the crown? And, further, if Cancer break out again before the healing of the wound made for its removal, and the recurrence be rightly attributed to incompleteness of the operation, is renewed disease equally traceable to residual fragments when two, five, or a dozen years elapse before recurrence? I may at once state that mere delay is not a sufficient reason for attributing recurrence of Cancer to the outbreak of a new and independent disease in an outlying structure which was healthy at the time of operation. It may be conceived to be quite possible that a residual fragment of the original disease should remain quiescent for years, 
since it is known that whole tumours sometimes continue as long without a perceptible alteration of size, and then suddenly enlarge at some one part. On removing such tumours, from the breast or from the parotid region, it is found that the torpid portion has been really cancerous, and probably scirrhous, whilst the character of the newly grown lobe is that of encephaloid ; cells predominating in the recent growth and fibres in the old.

In handling the subject before the Society I have to discuss the adequacy of the operation performed in the several cases which will be cited, and the inferences which should be drawn from the subsequent renewal of the local disease. If, on contemplating a series of such operations, an imputation upon their completeness is thought to be justified, then, so far as they go, they prove it superfluous to invoke the influence of the constitution, or a continuance of local growth which is assumed to persist in spite of a complete removal of the foregoing tumour, in explanation of its recurrence.

For displaying the method in which Cancer returns, it is requisite to select a region which may be readily observed, to distinguish changes in the disease which may be due to functional peculiarities of the part from such as are purely morbid, and, most especially, to take note of the very commencement of the renewed disease, before its early appearances are obliterated. At later periods of the case, when natural textures and scars are destroyed, and glands are extensively invaded, the lessons furnished by the mode of recurrence are lost. The organ most generally convenient for this inquiry is the mamma; and the conclusions deducible from the recurrence of Cancer in it are strikingly confirmed by observations made in parts which are less within the reach of common notice.

Taught without doubt by foregoing failures, our surgical ancestors adopted a method of operating which might well have been expected to prove effectual against a local recurrence of the disease. They transfixed the base of the mamma, and, 
raising it with ligatures, swept off the whole organ, together with all the skin that covered it. The proceeding had a barbarous appearance enough, but it was promising; and, if their knowledge of the disease had led the Surgeons of the time to adopt it before the skin was hopelessly infiltrated, they must have met with more success than they appear to have done. Postponing, however, all operation until the skin was brawny and covered with tubercles, and the deeper textures were involved without limit, they failed too often, with even such extensive cutting as they adopted, to comprehend the entire diseasè.

It was a mistaken kindness which led to a change of this mode of operating. Under the influence of a clergyman, who expressed what must have been a prevailing horror at such Amazonian surgery, the practice was changed to an incision in the integument, which was reflected in flaps and brought together again after the removal of the cancerous tumour. There could have been no diminution of suffering by this prolongation of the operation, and what was gained by it in neatness was lost in life. With the remains of the breast, as well as in their own texture, the flaps enfolded fragments of diseased substance, and Cancer soon reappeared. It was an operation proper to innocent tumours, which can be removed without the needless mutilation of extirpating the breast, but was quite inapplicable to cases of mammary Cancer.

In our own day various nodes of operating are practised. Sometimes the tumour only is removed; sometimes that segment of the breast in which the tumour lies is taken away with it; sometimes, with the intention of extirpating the entire mamma, the failure happens that a portion of it is unwittingly left behind; sometimes the breast is carefully removed, but the propensity of Cancer to extension in the skin is misapprehended, and for symmetry's sake a flap, which even includes the nipple, is preserved; and yet again, there being no definite plan in the mind of the operator but that of cutting wide of the tumour, portions of the organ itself are left behind. The consequence of this last method of operating may be at once apparent when, on examining 
the mass thus dug out of the centre of the breast, hard cancerous cords, continuous with the principal tumour, are found to have been cut across. Their outer extremities, prolonged to the margin of the breast, remain behind, and it is fortunate if the discovery be made before the wound is closed and the patient replaced in bed.

By the issue of operations thus conducted opinions on the nature of Cancer are always qualified and sometimes formed. When they have been extensive operations, there is an appearance of reason in assuming them to have been complete. But while, on the one hand, severe operations reduce the patient and favour the growth of Cahcer, on the other hand it must be considered that operations are not adequate merely because they have been large. A complete extirpation of Cancer could hardly be looked for except from a large operation for a recent and limited disease. For, whatever be the method of operating, the one important point, both for practice and theory, is to remove the whole. The least remnant is capable of growth, and may spring up into a new tumour with all the energy of the first. Recent experiments with acetic acid well illustrate the tenacity of life and the reproductive power of the minutest fragment of Cancer. By injections of that acid I have repeatedly, in the course of a few weeks, obtained the completest dissolution of a subcutaneous cancerous nodule; yet a few weeks later, in the place where, with the most careful touch, I could detect no remnant of the previous tumour, a growth has begun again, which in time became a nodule like the first. So subtle is the disease against which surgeons have to contend in their operations, and respecting which theories are framed out of its usual recurrence when removed.

This point appears to me a most important one, and one which would of itself suffice to excuse my thus occupying the attention of the Society. It is sometimes supposed that operations as at present conducted must be regarded as effectual, and that the recurrences so commonly ensuing cannot but be attributed to the nature of the disease. I venture however to aver that the difficulties of completely extirpating Cancer 
are much underrated by those who hesitate to suspect the perfectness of our operations. There is too often reason, without calling in the evidence of the microscope, to expect a return of the disease. Five times within a couple of months I have found supposed extirpations of Cancer to be demonstrably incomplete. I am far from wishing to convey the impression that the operators were themselves in fault in these cases. That which is to be gathered from the fact rather is that, when practised Surgeons meet with such difficulty in eradicating cancerous tumours, the subsequent recurrence is not due to any abstract quality of the disease.

The following cases are arranged with a view to illustrating the several methods of inadequate operating, and of throwing light upon one another. In some the tumour only was taken away; in some a greater or less proportion of the mamma was removed with it ; and in others, while the organ was extirpated, diseased tissues near it were left.

CASE 1.-Recurrent Cancer near two several wounds on the surface of the mamma.-I admitted into the Middlesex

Fig. 1.1

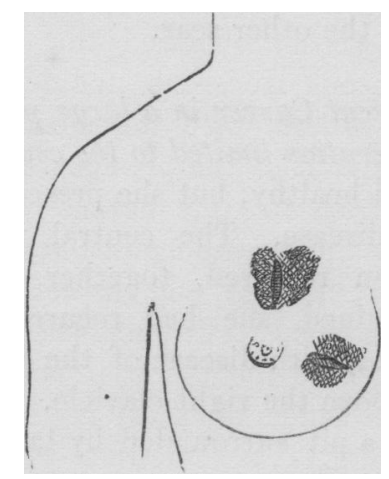

The central striped parts indicate the scars succeeding operations for the removal of separate cancerous tumours of the breast. The crossed lines around them show masses of Cancer which began to grow, in the one place before the healing of the wound, and in the other just after it. 
Hospital a tall woman, of about 38 years of age, having a recent scar and an unhealed wound on the surface of her right breast. The scar was already the seat of cancerous thickening, small, flat, but unequivocal. The skin of her chest was coarse, being covered with the white, yellow, raised or pitted little scars of a phthisical patient. She was quite free from pain in the chest; yet her respirations were forty in the minute, and Dr. Henry Thompson distinguished dullness at the apices of both her lungs.

She was married and had a family. Two superficial tumours formed in the right breast a year and a half ago; and nine weeks ago they were cut separately out of the breast.

It appeared certain from this immediate recurrence of the disease in and around the scar, and there only, that the tumours had been removed without the intervening and outlying fragments, and that nothing short of a complete extirpation of the breast could have comprehended them all. But, notwithstanding the possibility of still accomplishing that operation, I was led by her general condition and especially by the state of her breathing to dissuade her from submitting to it.

She went home, but during her short stay in the Hospital Cancer recurred in the other scar.

CASE 2.-Recurrent Cancer in a large portion of the right mamma after an operation limited to the centre of the organ.This patient looked healthy, but she presented a rather large amount of local disease. The central parts of her right breast having been removed, together with a cancerous tumour they contained, she had recurrent Cancer in the residue of the breast, with disease of the glands in the right axilla, as well as above the right clavicle.

The scar was in a pit surrounded by large tuberous elevations, making altogether a mass greater by half than the other healthy mamma, but crater-like instead of highest in the middle. One of the great lobes was softening, and the thin skin covering it was of a dark purple colour. Except at this 
softened lobule and the scar, there was no adhesion of the tumour to the skin, and it could be moved with some freedom

Fig. $2 .^{1}$

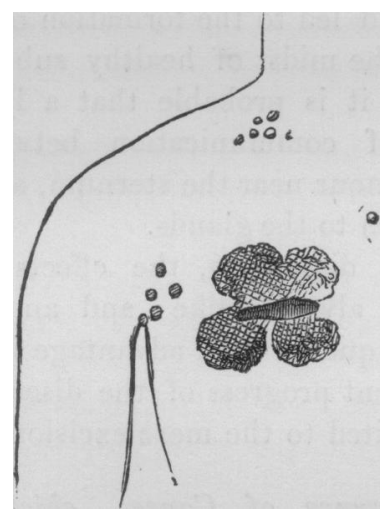

over the pectoral muscle. Apart from the principal mass, and beneath the skin above and within it, could be felt a movable hard nodule, larger than a cherry stone. Such a nodule, if found by itself at a time long subsequent to the extirpation of the breast, might be supposed to be an independent and spontaneous disease ; but its growth simultaneously with, though somewhat apart from, the chief recurrent disease demonstrates a connection between them, which is of importance in judging of the source of a similar nodule when observed alone. See Case 10.

In Cases 1 and 2, the disease recurred in the textures which had been divided in the operation, and spread in every direction from beneath the scar. The return in the second case was not indeed so rapid as in the first patient, several

The oblique stripes in the middle of the disease indicate the position of the scar, around and beneath which are lobes of Cancer, somewhat inaccurately represented. Scored circles in the right armpit and the neck correspond to cancerous glands. A little above and internal to the inner and upper lobe is one small subcutaneous isolated cancerous nodule. 
months having elapsed before any renewal of the growth was recognised. In a couple of years, however, the remnant of the mamma was all involved in it, as well as the glands both below and above the clavicle; whilst in its increase towards the sternum it had led to the formation of an isolated cancerous nodule in the midst of healthy subcutaneous tissues. From later cases it is probable that a lymphatic was the unseen channel of communication between the primary disease and this tumour near the sternum, as was certainly the fact in its extension to the glands.

Though equally disastrous, the effects of this mode of operating are not always alike; and another case of the same kind may he quoted with advantage in this series, as it illustrates a different progress of the disease after an operation purposely limited to the mere excision of the tumour.

CASE 3.-Recurrence of Cancer, chiefly in the axilla, after a limited operation for scirrhus of the mamma.A scirrhous tumour was cut out of the right breast of a lady by a horizontal incision above and outside the nipple. Fifteen weeks afterwards she came to England, and I saw her with my colleague, Mr. Hulke. The scar was a model of evenness and neatness, and the form of the breast was scarcely altered. There was also no distinct hardness or tumour beneath the scar, though there may have been some thickening, and the remainder of the mamma was tender. Some of the glands, however, having lately enlarged, formed a cluster of firm tumours in the right axilla. The highest limit of the disease in the armpit was a little uncertain, but there was no Cancer above the clavicle.

It was recommended that the axilla should be opened, and that, if the whole of the diseased glands could be taken away, the breast also should be removed; but that the latter operation should not be proceeded with, if diseased glands were to be left behind the pectoral muscle.

It being found impossible to clear away all the glands, $\mathrm{Mr}$. Hulke forebore from amputating the breast. He has since 
informed me that, though the disease in the mamma proved to be cancerous, it did not afterwards much increase; in the axilla, however, it continued to grow and to fill that cavity, until in less than a year afterwards she died.

Though neither from beneath the areola nor from the surface of the mamma is Cancer extirpated by the removal of the tumour only, it may still be questioned whether it is needful to sacrifice the whole breast when the tumour implicates but one edge of it. The disease then has such an appearance of isolation, and the remainder of the breast is so perfectly soft and healthy, that Surgeons cannot but be tempted to remove the diseased edge alone, or at most that segment of the organ in which the tumour is seated. Especially if the disease be recent, is there danger that this method of operating may be adopted. Indeed it cannot be denied that such operations are sometimes followed by an interval of health which, in contrast with the earlier recurrence of older and more diffused Cancers, the Surgeon may deem satisfactory. But that mode of judging the result is surely fallacious. The question should rather be thus stated : if persons so operated on survive a given number of years and then suffer a local recurrence of their disease, how much longer exemption would have followed the removal of the whole breast? Surgeons who thus operate do less for their patients than the case allows, and niss the best hope we know of permanent cures. It is just in these cases of recent and limited tumours, that the total extirpation of the mamma obtains its fullest reward. Whereas, if the removal be incomplete, it is in the breast which should have been taken away, or in a structure closely connected with it, or most commonly in the very site of the operation, that a fresh outbreak of disease proves the error which has been committed. Were this method of operating right, there should happen cases in which Cancer arose in a distant organ, while a portion of a mamma from which a primary Cancer had been removed remained exempt from the disease. I have not met with such cases. 
CASE 4.-Recurrent mammary Cancer in the site of a former operation limited to the outer portion of the breast.A patient, æt. 46, rather stout and pale, was brought to me, from the outermost edge of whose right breast a cancerous tumour had been removed fifteen months before, and the disease had returned.

The margins of the breast appear healthy above, within, and below. The central and outer parts are hard, broadly depressed, and covered with adherent pitted skin, in which the

Fig. 3.1

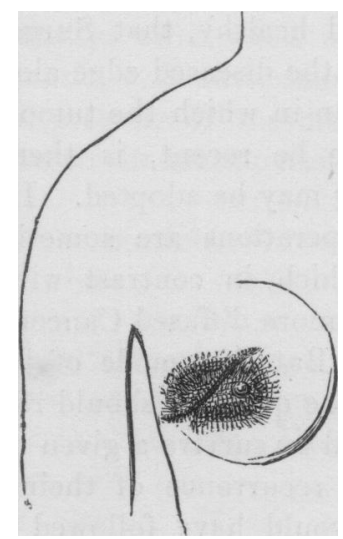

nipple lies, shrunken and drawn in. Immediately above the depressed surface the skin is soft and thin, but it is thickened chiefly, if not only, by œdema, over the whole of the lower part of the breast. The broad mass of Cancer occupies the entire depth of the breast, and adheres to the muscle beneath. The scar of the operation is linear, and crosses the outer part of the disease obliquely from without, upward, and inward ; two inches at either end of it are perfectly soft, healthy, and unadherent to the textures beneath it; the middle part, though still discernible, is mixed up

1 The diagram represents a mass of solid Cancer, involving the central and outer parts of the right breast, together with the nipple and the middle of a long oblique scar. 
with the diseased mass. Some of the lower axillary glands are large and hard.

This disease becoming painful, and being apparently capable of removal, I repeated the operation. The soft healthy upper skin I cut near the diseased; the lower, which was odematous, at about an inch and a half from the diseased. The scar was divided in the two parts where it crossed the long oval incision. I turned the flaps off from the breast, which $I$ detached first at its edge, and then removed in one mass with an adherent piece of the muscle and with the diseased axillary glands. Though nearly certain that the skin at the lower part of the incision was œdematous only and not cancerous, I touched its edge with the solid chloride of zinc. The wound was made in large lobules of fat, and before closing it, I sponged it freely with Zinc. Chlor., grs. 40 to an ounce of water.

The wound healed in thirteen weeks, and the patient went to Brighton, but with doubtful thickening near the scar.

Four months after the operation I saw her again, and found her in the fullest health. The disease had resumed its growth, and formed a low nodular hard tumour on the side of the chest, in connection with the axillary end of the second scar, and with thickened skin just below it. Not being freely movable, it was probably also attached to the subjacent muscle. It was three inches broad at the scar, from which it extended three inches upwards into the axilla, and downwards two inches. The integument above the scar was soft and healthy, but that below it was tense, partly from œedema and partly from complete contraction of the wide wound to a linear cicatrix.

With so great an improvement in her general condition, it was impossible to attribute the recurrence to an increase of constitutional disease. There was now none which could be discerned amid healthy functions, healthy look, and healthy feeling; yet the local tumour had returned with a vigour greater than after the first operation. Neither was the recurrence diffused, either in the body, or over the mammary region, or even throughout either scar; but it was limited to

voL. $\mathbf{L}$. 
just those portions of skin and muscle and abundant fat which had most nearly adjoined the disease, and being suspected had been in part taken away. The result shows an error in my operation on the side of defect, and also, by a strictly local recurrence, a local disease.

CaSE 5.-Cancer recurrent in the breast beneath the scar of an operation.-A very stout woman, æt. 45, consulted Mr. John Scott, and was sent by him to me. At the inner and upper margin of her left breast was a circumferential scar, of four or five inches in length, with marks of needle punctures beside it. The scar and skin were soft, but the former was attached at one part to a tumour in the substance of the breast. The tumour was as large as a goose's egg, uneven, hard, covered with breast tissue and adherent to it. The mamma being very large, the tumour did not reach the nipple, which was not retracted. In each axilla was an indistinct nodule, feeling like an enlarged gland, and that on the right side was larger than that on the side of the diseased breast. There was no glandular disease in the neck.

The patient had been a healthy woman : she had had five children, the youngest five years ago. All her labours were difficult, and the last child was perforated. The tumour had been removed two years, but eight months ago it reappeared.

I advised removal without delay, and she returned to the country to have the operation done at home.

The lesson furnished by this case is the same as that of those which precede it. Recurrence was neither constitutional nor organic, nor regional, but that of an incompletely extirpated tumour.

CASE 6.-Cancer, recurrent after a partial removal of the breast. - A thin and pale sempstress, æt. 54, had a long vertical scar, extending almost from the clavicle to the right nipple. The highest part of it was about an inch in breadth, soft and healthy; the lowest part was narrow and somewhat sunken in the breast. That organ itself was flaccid and thin, 
and it appeared to be healthy; but on its surface, an inch outside the lower extremity of the scar, was a firm cancerous

Fig. 4.'

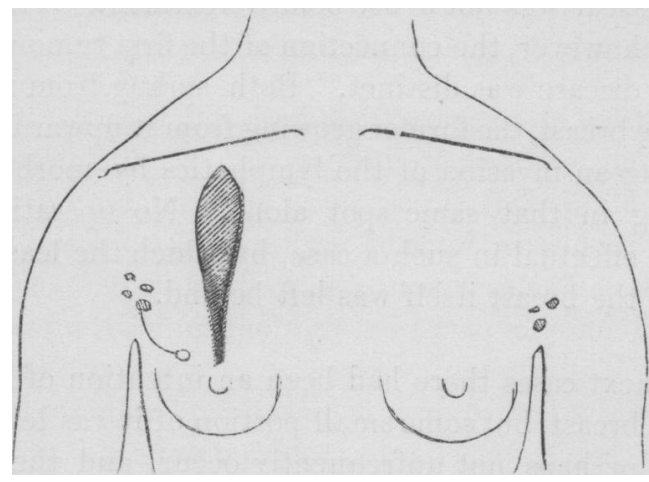

tumour, of the size of the end of the little finger, movable, subcutaneous, not distinctly attached to the breast or to the scar. Thickened lymphatics passed outwards from it under the pectoral to some enlarged and firm axillary glands. There was also a slighter enlargement of glands in the left armpit; the left mamma was healthy.

A tumour at the upper part of the right mamma attracted the patient's notice several years ago. It was at that time of the size of a walnut, but as it caused no pain and did not distract her from her hard work, she paid it no further atten. tion, and only afterwards remembered it. When, however, seventeen months ago, it had grown upwards in the form and size of a pear, and nearly reached the collar-bone, it was removed, without the breast.

I first saw the patient after the tumour and adjoining tissues

I A vertical scar, represented by oblique lines, extends from the upper part of the right breast nearly to the clavicle. The original tumour began in the mamma, and the recurrent tumour an inch on the outside of it. The line drawn from the latter tumour to cancerous glands in the axilla is intended to represent thickened absorbents, which may be easily felt in the patient. The glands in the left armpit are likewise somewhat enlarged. 
had been freely injected with acetic acid, and found the lowest part of the scar thickened, and apparently connected by tumid breast tissue with the tumour. Mr. De Morgan, under whose care the patient had come, informed me that the lowest part of the scar was not dense before treatment. Without this condition, however, the connection of the first tumour and the recurrent disease was distinct. Both sprang from the upper edge of the breast, the former growing from it upwards, and the latter being an invasion of the lymphatics by morbid matter originating in that same spot alone. No operation could have been effectual in such a case, by which the least morbid portion of the breast itself was left behind.

In the next cases there had been an intention of removing the whole breast, but some small portion of it was left behind. This may perhaps not unfrequently occur, and the remnant of the mammary substance may gradually wither and be absorbed. But I have had no opportunity of ascertaining the fact, and whether such involution be or be not usual after incomplete removals of the breast, the following cases show that event to be not invariable. Now if, when a portion of the organ has been left, the renewed disease begin in distant or in various parts of the mammary region, its reappearance may be held to be uninfluenced by the method of operating; whilst an outbreak of Cancer in the remnant of the mamma, and its dispersion from thence only, prove at once the exclusive connection of that part with the foregoing disease, and the correctness of the theory of its local origin. Such a fact indicates clearly the propriety of a complete removal of the organ in which the disease arose, and establishes the theoretical inference drawn from previous cases in which a larger portion of it was designedly left.

CASE 7.-Cancer, probably recurrent, in a small internal portion of the mamma left in a previous operation.-A patient came under my care in 1866, from whom the right breast had been removed a couple of years previously. I found a vertical soft broad scar on the right pectoral 
muscle, and towards the sternum a subcutaneous firm prominent movable tumour of twice the size of an almond. From its position over the costal cartilages, on a horizontal level with the middle of the scar, and distant from it about half its length, or the radius of the breast, there could be no doubt that the tumour was a portion of the inner edge of the breast, which had become thickened. There was no other disease on the surface of the chest or in the axilla, but the patient was uneasy about the tumour, and complained of various pains about the right side of the chest. She was out of health, and looked phthisical, and whilst under my observation she had a bilious attack and some painful hæmorrhoids.

As the tumour itself, though dense, was not distinctly hard, nor was it the seat of pain or tenderness, I could not be certain that it was the cause of her ailments. After watching it therefore for two or three months, and finding that it did not increase, I let her go away. Nevertheless, it now appears to me by no means certain that her uneasy feelings may not have been due to the existence of Cancer in the thickened remnant of the mamma, if not also to its extension through the intercostal absorbents to glands within the chest.

CASE 8.-Recurrent Cancer in a remnant of the mamma, infecting the subcutaneous lymphatics and the axillary glands, but not the scar.-A lady, aged about 40, was brought to me two years after the removal of her right breast. The operation had been a very thorough one, and the scar was large. It was thin, pliant, and movable on the subjacent textures. Beneath the skin at its inner edge was a three-cornered hillock, shaped like a cashew nut, precipitous outward, bevilled towards the sternum, and about an inch and a half in its vertical measurement. Its outer surface was firm, and the slightly thickened skin covering it showed enlarged pores when lifted in a fold. The mass appeared to be the innermost edge of the mamma partially infiltrated with Cancer. There was no disease in or beneath the scar, but along the 
upper and lower borders were several cancerous nodules, of the size and slipperiness, and almost of the hardness of peas.

Fig. 5.1

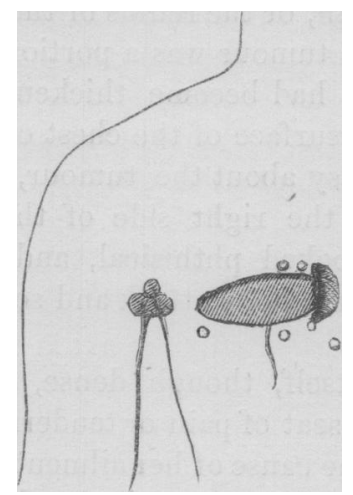

The skin covering these nodules had no adhesion to them. Behind the edge of the pectoral muscle lay four enlarged and hardened glands, closely adjacent but not adherent to one another. A cord of lymphatic vessels led upward from the highest of them, and was lost behind the pectoral. No other gland could be felt in the armpit, and the space above the clavicle was healthy. There was no disease in the left mamma. The recurrence of these growths had not much attracted the notice of the patient, who, however, had spoken of pains for about six months.

There could not be a more definite illustration of the local characters of Cancer. By an extensive operation the original tumour, with the breast and a broad covering of

\footnotetext{
1 The oblique stripes and the line depending from them represent the scars remaining after the removal of the right mamma. Internal to the scar is a fragment of the mamma, cancerous; parallel with the scar, both above and below it, are subcutaneous cancerous nodules, believed to be connected with lymphatics, by which also portions of the disease have been conveyed from the principal tumour at the inner end of the scar to the glands, represented by scored circles, in the armpit.
} 
skin and superficial absorbents, had been removed, and there was no recurrence in the scar. A small part of the inner edge of the mamma having, however, been left, the disease had continued to grow in it. This portion now constituted a primary tumour, and from it, as a new centre, the growth disseminated itself in the usual way, passing by the lymphatics to the nearest glands in the axilla. But, there being no lymphatic canals in the scar, which was in the direct line to the armpit, the morbid matters had taken a circuitous course above and below it, and had formed small subcutaneous nodules, such as we are accustomed to find by dissection in the track of lymphatic vessels. Full and probably thickened absorbents in the axilla showed that the disease was already passing in the same manner into yet higher sub-clavicular glands. Moreover, from the existence of one or two nodules lower down on the chest than the area of the operation, and out of the line of lymphatics between the principal tumour and the scar, there were indications that the disease was ready to extend in other directions than towards the armpit.

In the next series of cases the entire breast appears to have been removed. It is of course difficult to secure absolute certainty of the removal of all the mammary structures, as the precise limits of the breast are ill-defined, and vary much in different persons. But there is every probability in the following cases that the operation, so far as the breast is concerned, was complete.

CASE 9.-Recurrent Cancer in skin and cicatrix, infecting the subordinate lymphatics and glands. - In December, 1865, Mr. Square, of Plymouth, removed the right breast of a slender woman, aged about 44, and subsequently by a second operation took away a small fragment of apparently recurrent disease below the innermost end of the horizontal scar. On a further return of the disease he recommended the patient, who had come to reside near London, to apply to me, and I saw her in May, 1866. 
I found that the right breast had been completely removed, and in its place was a thin and nearly horizontal scar, movable over the right pectoral muscle, and forked at its inner end. About the lower of these branches of the scar there

Fig. 6.1

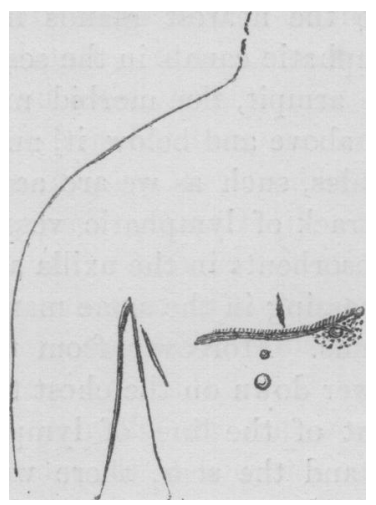

was some shooting pain, and a little enlargement of the pores of the skin. Low in the right axilla was a globular, firm, tender, slippery gland, as large as the end of the little finger. Three weeks afterwards there were two enlarged glands in this armpit, and I advised her to have them removed. After a further delay of a month this was done. But before the autumn she returned to me with a hard peashaped nodule fixed in each of the inner branches of the scar, and with two similar but perfectly movable subcutaneous tumours a little below the middle of it. The axilla was now healthy.

The explanation of this occurrence appears to be that some of the original disease remaining in the skin at the inner end

1 In the place of the right mamma is a horizontal scar, forked at its inner end. The dots represent slightly thickened skin; the circles show nodules of Cancer, the inner two being cutaneous, the lower two subcutaneous; the line in the armpit indicates the scar left after the removal of some cancerous glands. 
of the wound multiplied at first in that structure, and afterwards entering a lymphatic, began to grow on reaching the axillary glands. The removal of these glands checked the ascent of the disease to higher parts of the axilla, but did not lessen the supply of morbid matter from the sternal end of the scar. As this increased, it assumed the form of little tumours, and continued at the same time to fill the lym. phatic channels connected with it. But the axillary extremities of these vessels having been closed by the last operation, the morbid matters accumulated and formed tumours in their course. The removal of lymphatic glands, and their complete destruction by Cancer, thus produce equivalent effects. They dam up the current in the afferent lymphatics and cause its accumulation in tumours, so long as the primary texture continues to furnish cancerous matter. Accordingly it now appears to me that my operation of removing the glands alone was not an adequate operation. More importance has since attached to the merely thickened skin ; and in the subsequent progress of the case it has been shown that that small factor of morbid material, though attracting little attention at first, should likewise have been removed.

Into each of the four nodules I injected acetic acid in the manner proposed by Dr. Broadbent, and found them in three or six weeks either much reduced in size or no longer perceptible. But in the course of a couple of months each tumour had grown afresh, those which had disappeared arising first as a soft swelling, and then regaining their former hardness and globular shape. After this event had repeatedly occurred, I injected each nodule somewhat freely with undiluted acetic acid. The outer two nodules, which were entirely subcutaneous, subsided; the inner two, being implanted in tough scar and thickened skin, sloughed. The further effect of this destruction of the residual disease, on which depended the infection of the lymphatics and glands, is not yet manifest.

CASE 10.-Recurrent Cancer near the inner and the uppermost extremities of the scar, after an extensive operation 
for the removal of the right breast and its axillary glands.In 1859 I removed the right breast of a patient, who had fifteen months previously discovered in it a cancerous tumour of the size of a nut. The operation was an extensive one, and having taken away the axillary glands with much tissue, I put a drainage tube through the back of the armpit. The patient recovered health and was perfectly well for four years, when she began to have pain along the nerves of the right arm. In the following two years that arm became cedematous and weak, and she carried it in a sling. On her applying to me again six years after the operation the arm was much swollen and weak, but the pain in it had diminished. The scar was wide and adherent to the chest, but both it and the adjoining structures were perfectly healthy, and I could discern no tumour whatever in the axilla. Neither was there any swelling above the clavicle, but as she could not turn her neck freely, I examined it closely and found that, with

Fig. $7 .^{1}$

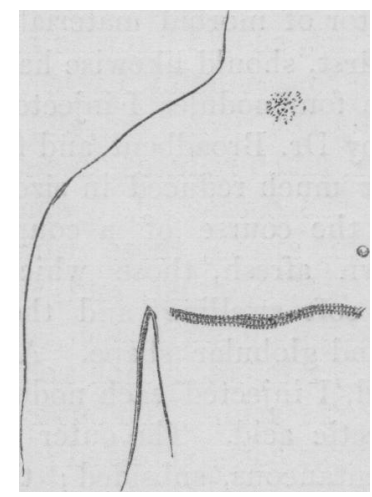

1 The scar, much too small and regular in form, as well as deficient towards the axilla, is indicated by an obliquely striped line. A cluster of dots in the neck points out a small spot at which the skin is dimpled over a small, fixed, cancerous mass about the brachial plexus. This was discovered six years after the operation, though it may have been growing from the fourth year. Above and within the inner end of the scar, as in Case 2, a small, isolated, subcutaneous nodule, formed seven years after operation. 
the head inclined to the left, the right scaleni dragged upon a tough spot near the right brachial plexus, over which the skin, without being thickened, was a little adherent. The dense substance in this spot was not large enough to form a swelling.

After another year the œdema had under treatment almost subsided, and the arm was without pain; but it was so weakened that it might be said to be partially paralysed. Sensation was unimpaired, and the fingers could be moved, but there was no power over the shoulder-joint, elbow, or wrist, and the limb could not be raised into the sling without the help of the other hand. No increase had taken place in the hard thickening beside the lower cervical vertebræ, and the skin was even a little loosened from it, but the scaleni were more drawn. Examining the chest again, I now found one cancerous nodule beneath the skin over the sternal end of the right second intercostal space. It was a little larger than a cherry-stone, and was situated so far from the scar as to be out of the area of the wound made in the operation. It was moreover quite isolated, no remnant of the breast being anywhere perceptible. In December, 1866, I injected the nodule and the dense substance at the root of the neck with diluted acetic acid, and found both of them diminish. The skin became looser in the neck, and that over the shrunken nodule on the chest sunk in and showed a visible dimple.

This case is perhaps an extreme instance of the uncertainty of the distinction between reawakening of a long dormant residue of living Cancer, and a fresh outbreak in the region, as distinguished from the organ, first attacked. The first recurrent Cancer, which was in the neck, appeared, from its position, to be unconnected with the original disease. For whilst in all the former cases recurrence was first observed about the scar, and the subsequent disease involved the axillary or the supra-clavicular glands, in this case it arose amongst the nerves, and was first discovered in relation with the scaleni, the brachial plexus, and the transverse process of the lowest cervical vertebra. The form of the growth when first observed did not indicate an origin in a gland, and 
it was not till a year afterwards that $I$ found the nodule above the scar. These difficulties are, however, only apparent. The form of recurrence could hardly have been usual after so extensive an operation, and in a case in which the rate of the subsequent growth of the disease appeared to lengthen. Had the first renewal taken place over the pectoral, the breadth and adhesion of the scar would have ob. structed its course by the lymphatics towards the axilla, and diverted it over the clavicle to the glands in the neck. There would thus have been no advance of the disease to the armpit, even had any glands been left there, which could have received it. The only other glands which could have been infected by this superficial recurrent growth, namely, those above the right clavicle and in the left armpit, were healthy. It appears clear, therefore, that the cancerous growth about the brachial plexus could not have arisen in continuity with the small subcutaneous tumour near the sternum; and the course of the symptoms associates it with some fragment, which was left among the nerves in the highest part of the axilla, and which began to grow at the time of the first pain in the arm.

Though the disease first noticed was in the neck, and the nodule over the chest was not found till a year afterwards, it is difficult, though it might not be unreasonable, to regard the latter as the primary. For, when glandular disease is the first to attract attention, it is not uncommonly excited by a primary Cancer of so small a size as to be discernible only at a later period of its growth. Inasmuch, however, as the cervical disease does not appear to have been glandular, there is no need to suppose any such exceptional explanation, but to connect it with an axillary portion of the original disease. I add another of my cases which seems to bear out this opinion.

CaSe 11.-Probably recurrent Cancer in the axilla six years after an extensive operation. - Emily $\mathrm{N}$-, æt. 45, came under my care in 1855, having had tenderness in the left breast for twelve months, and more recently tenderness and shooting 
pains in the right breast and whole right side, with headache and other symptoms, which appeared to arise from the excessive use of tea, and which subsided under treatment.

The breasts were very large. The left was natural, except at the upper part of its axillary border. At this part there was a slight pit, the skin was thickened and adherent, and the mammary tissue was found on superficial examination to be nodular and a little hard, but no distinct tumour could be felt. At the corresponding spot in the other breast, where the axilla and breast met at the edge of the pectoral muscle, was a similar pit, without thickening of the skin. The left nipple was not retracted; the left axilla appeared to be healthy; the left external jugular vein was more prominent and larger than the right.

November, 1855.-I made an incision into the thickened part of the left breast, and, finding there a small but distinctly scirrhous tumour, I removed the whole organ. The wound was large, but few vessels bled.

April, 1856.- She complains of her right breast, but there is no growth in it.

June, 1858.-There is no return of the disease; the cicatrix is sound, and her health is good. There is no tumour in the right breast, to which, however, her attention seems much directed. She has a few sebaceous tumours on the scalp.

June, 1863. - She has applied to be a Cancer out-patient, and has been kindly sent by Mr. Nunn to me. She was stout and apparently in good health, but her breath was short, and she had severe intermittent tingling or scalding pains in the left fingers, or in parts of the left arm which she indicated with great precision. These had been first noticed in November, 1861, but they had lately increased.' She described them as like the burning of molten lead or boiling water, and her only relief, until they went off, was obtained by soaking the limb in hot water or flannels. The scar was healthy and perfectly loose. The left side of the neck, the whole scapular region, the pectoral and the arm, were odematous, moderately but plainly so. There was no trace of disease in any axillary gland.

November, 1863.- The pain down the arm continues 
intense; the movements of the shoulder are restricted, but the arm is little swelled. The pectoral muscle is raised, and the hollow of the axilla is deeper than before. At its highest part is a small ulcerated surface, not distinctly cancerous. Two or three glands above the clavicle are enlarged and hard.

Her sufferings were mitigated by the application of morphia to a blistered surface on the left side of the neck, and by subcutaneous injections of the same salt. They continued, however, to be very severe, and towards the end of her life her breathing became peculiar and difficult, apparently from some affection of the left phrenic nerve by the disease in her neck. This, however, could not be verified, as, on her death, which took place in the spring of 1864, permission to examine her body could not be obtained.

Notwithstanding the long period of six years which elapsed between the operation and a renewal of symptoms in this case, I cannot doubt that I had incompletely extirpated the disease. Some cancerous invasion of parts behind the left clavicle, and therefore beyond reach, must have already occurred when I removed the breast. Though much protracted in its rate of growing by the loss of the primary tumour, the disease proceeded in one direction just as if it had remained in continuity with the mammary Cancer, involving the subordinate glands only and the adjoining nerves. In every other direction it ceased.

A longer interval than any yet given may elapse between the removal of a cancerous tumour and the local return of the disease. So late indeed may be the recurrence that it is not surprising to find it regarded as the outbreak of a new and independent disease. Yet in all but its delay the recurrence is precisely similar to that which has been noted in previous cases.

CASE 12.-Cancer, recurrent at the border of the scar more than nine years after the removal of a recent scirrhus of the breast. Further exemption from the disease, already known to have lasted for seven years, after a second operation.- 
Sarah H-, æt. 47, was one of a family of six sisters, five of whom and their mother suffered from Cancer of the left breast. The tumour in the patient Sarah $\mathrm{H}$ - was removed by Mr. Arnott in December, 1846, four weeks after it was discovered. It was about the size of the last joint of the thumb, and situated around the nipple, which, however, was not in the least retracted; and it was an example of genuine stony Cancer. From the time of the operation she enjoyed good health, until the beginning of 1856, when she perceived a return of the disease in the cicatrix. In April of that year there was at the axillary border of the cicatrix a hard tumour of about the size of a small pigeon's egg, adherent to the integument, though free over the surface of the chest. In the axilla and above the clavicle were some enlarged but not indurated glands. This lump, together with four axillary glands, was extirpated by Mr. Shaw, and both to the naked eye and under the microscope the whole was found to be made up of well-marked somewhat soft scirrhus.

It is hard to include among inadequate operations one which was for so many years effectual. Yet, if the recurrence were due to delayed growth of some fragment of unhealthy tissue which was left at the time when the principal mass was removed, it must be so included. That this was really the case appears proved by the position of the renewed disease. Not in a distant part, not in an internal organ, not even in the remaining breast, but in close proximity to the scar, and on that side of it which was in the direction of natural dispersion of the first Cancer towards the axilla, this recurrence connects the second with the primary tumour. The remarkable continuance of health after the first operation moreover dissociates the disease from any separate accumulating constitutional ailment; and it further shows the dependence of internal Cancer, and even of the infection of the second breast, upon a certain amount or mass of the local tumour, which this early and admirable operation reduced. In the absence of growing material, diffusible from the primary Cancer, the whole of the remainder of the body continued healthy. 
Case 13.-Recurrent Cancer below a transverse scar, after the removal of the left mamma and its axillary glands. Long abeyance of the disease above. the scar, especially in a

Fig. ${ }^{1}$

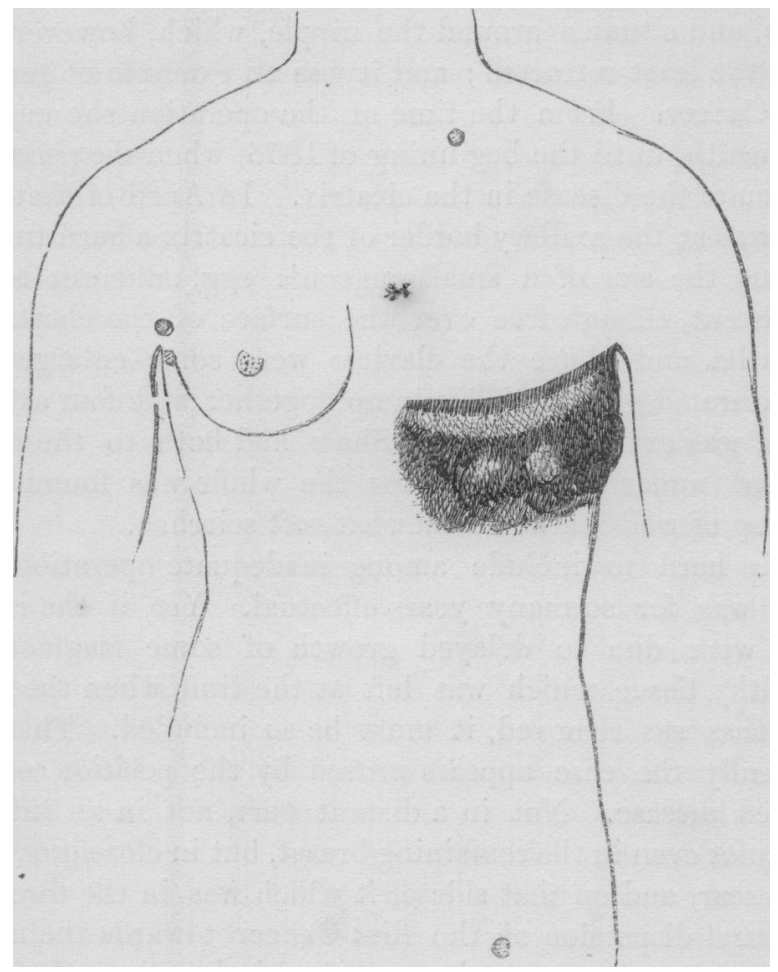

1 The diagram represents a large mass of Cancer abruptly limited at its upper part by a scored transverse line, which indicates the position of the scar. A striped circle in the neck marks a cancerous gland, diseased at the time of the operation, and unaltered afterwards (notwithstanding the exuberance of the growth below the scar) until in a later stage of the case the disease broke through the scar and flooded the healthy textures on the wall of the chest above it, as well as the glands in the neck. Striped circles in the right armpit and the left groin show the position of glands to which disease was conveyed by collateral lymphatics from the recurrent Cancer on the left side of the chest. 
cervical gland, which was enlarged at the time of the operation, and which increased so soon as the disease below had destroyed the scar.-The next diagram represents a case in which $I$ removed a large cancerous left breast with the axillary glands, deliberately leaving a solitary, enlarged, globular and movable gland behind the sterno-mastoid muscle of the same side. Though it was my intention to remove the entire mamma, and it is my belief that I did so, yet the disease speedily returned below the scar. The course of the recurrent disease, and that in the cervical gland, which were thus separated by the scar, presented a singular and instructive contrast, the conditions of which were ordinary, and intelligible without reference to the constitution.

The recurrent mammary disease increased below the scar, and in the course of three or four months became a large mass of great prominence and breadth, abruptly separated by the scar from the healthy structures. Immediately above the scar the integument was level, soft, pale, thin, movable on the pectoral, and indeed perfectly healthy. Immediately below the scar the disease rose in a precipitous swelling, distended with odema and venous blood and covered with turgid skin. The gland behind the sterno-mastoid remained no larger than it had been before the operation. Though in the same constitution, and nourished by the same blood as the disease which was so active below the scar, it continued absolutely quiescent. After some weeks more, two glands became cancerous in the right axilla, though no tumours could be felt in the right mamma; and it appeared that the great mass of disease, which now covered most of the left side of the chest from the scar downward, and was approaching the mesial line, had infected the glands of the right armpit through collateral lymphatic circulation. Meanwhile the gland behind the left sterno-mastoid remained unaltered. At length, however, the disease passed through the scar, and began to form small cancerous nodules in the healthy skin above it. The effect of this upon the glands above the clavicle was immediate. In three days they were perceptibly increased, and that dis- 
eased one behind the left sterno-mastoid, so long unaltered, now distinctly enlarged. Three weeks later it had nearly doubled in size, and it was united in a dumb.bell shape with an adjoining gland which, though previously imperceptible, had grown to be nearly equal to it. From that time disease spread rapidly both above and below the clavicle. The scar became obliterated in the growing and ulcerated mass, and when the patient died, the superficial collateral lymphatic circulation had conveyed some of the morbid material to one of the glands in the left groin.

The inference from this striking case plainly is, that the extension of the disease was that of a local ailment only. It spread by continuity of the morbid material : where tough scar interposed, its progress was arrested; existing disease, if not continuous with the principal local mass, was in abeyance, no assumed ailment of blood stirring it to the least activity; and there was a total exemption of other parts of the body from the disease, except such as were in communication with it by lymphatics. To search in the constitution for the source and control of an ailment so definitely ruled by local conditions appears superfluous. This, however, may be added in explanation of the peculiarities of the case. The cancerous cell elements formed an unusually small proportion of the substance of the disease. They were mixed with an abundance of a thick liquid, having some resemblance to the cream of milk; and by this, whilst the transfer of the cells from place to place was facilitated, their destructive action on the natural textures and their multiplication when transplanted were probably hindered. This curious association with a growth of Cancer appears to have been a functional disturbance, and to have been essentially an excessive secretion of milk. From another case which has occurred to me there appears reason to think that the tendency to it may be taken up and continued by textures adjoining the breast, though the product of the undue activity of those textures is of course not milk, but such a liquid as their construction allows them to exude.

Seeing that the disease was so distinctly limited, it may be 
asked whether a more extensive operation might not have availed to eradicate it. My own impression is that this would have been improper. The operation which was performed was a large one, and during the time when the local recurrence was limited to a few small tumours, the general condition of the patient did not admit of the operation being repeated. The case illustrates none the less the consequences of a partial removal of the disease, and a continuity of the morbid growth over a very wide area of the body.

The disastrous result of leaving the skin and nipple when the breast is removed is illustrated by the next case.

CASE 14.- Recurrent Cancer after an operation in which the breast was removed, but the skin covering it and the nipple were retained.-A patient came to me in much suffering from recurrent Cancer and dyspnœa. In the place of the right breast was a shallow hard-edged ulceration, measuring horizontally $4 \frac{1}{2}$ inches by $2 \frac{1}{4}$ inches vertically, and surrounded

Fig. 9.

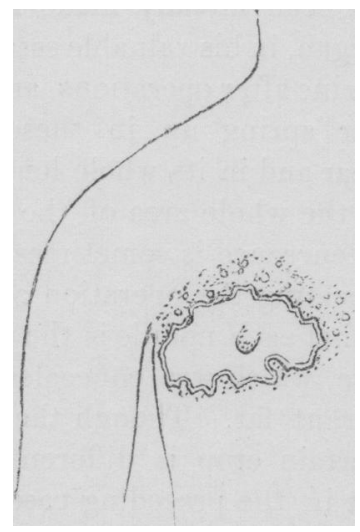

1 In the centre of the diagram is the cancerous nipple, surrounded by a large ulcer, which has destroyed the remainder of the flaps of skin, and beyond the sinuous edges of which the thickened integument, with pores enlarged and beset with cancerous tubercles, is indicated by dots and small circles. 
by dense adherent skin, which was thickly studded, especially above, with cancerous tubercles. Isolated in the midst of the almost level ulcer was the nipple, also diseased. The ulcer was in an irritable state, and there was bright inflammatory redness of the integument down to the lower margin of the chest.

The patient was an Irish washerwoman, who appeared to be 30 years of age, but supposed she might be 40 . Her history was that the right breast had been removed eight months previously for Cancer of ten months' standing, and by an operation in which the nipple was not removed, but the skin covering the breast was raised in a flap and then laid down again upon the pectoral muscle. The wound healed quickly, but not completely; it soon reopened; all the flap ulcerated except the nipple ; and on her applying at the Middlesex Hospital every structure in the area of the wound made in the operation, and for some distance around, was cancerous.

There is one mode of recurrence of Cancer almost exclusively peculiar to persons having much subcutaneous adipose substance. It has been already made known by my colleague, Mr. De Morgan, in his valuable essay on the use of solutions of chloride of zinc after operations, and in recent wounds. Nodules of Cancer spring up in these patients in close proximity to the scar and in its whole length, or (which is an equivalent fact) in the whole area of the wound made in the operation. The recurrence is sometimes so speedy, and the appearances on repeating the operation are so decisive, as to leave no question that each nodule is the product of a residual fragment of disease, which was concealed at the time of the operation in exuberant fat. Though the texture holding the seed of the too certain crop is different, the occurrence is identical with that in the preceding case, in which a multitude of germs were folded back upon the wound with the flap of skin and nipple. It is not every amputation of a mamma beset with much fat, which is followed by so diffuse a growth of Cancer; and there is reason to think that great care in taking away this superfluous substance might be attended with a result as satisfactory as that in Case 10. 
Though the foregoing cases may not illustrate all the phases of recurrent Cancer of the breast, they appear to bear with a decisive uniformity on the questions respecting the nature of the disease. It is possible that there may be methods of recurrence which would favour other conclusions than those I am about to draw : but as my cases have not been selected to prove an opinion already formed, I am satisfied that they represent the usual occurrences. They are also in accordance with examples of Cancer, which have been demonstrated to recur in the same manner in other organs. The following are the conclusions to which my cases lead.

Local recurrence of Cancer after operations is due to the continuous growth of fragments of the principal tumour.

Such recurrence may take place also in a residual part of the organ, respecting which it cannot be asserted that it was cancerous at the time of the operation.

Such recurrence may further happen in a structure adjoining a completely extirpated breast, and on a comparison of cases may be held to be produced by disseminated fragments of the original tumour.

The recurrent Cancer is subject as well as due to local conditions, and especially adapts itself to the distribution of the absorbents. Its continuity with the first tumour may be traceable over half the chest, the pleura, and the glands: from the neck to the loin or the inguinal region, and possibly also to the liver.

After the removal of a portion of the breast, the recurrent Cancer does not involve the remainder of the organ indiscriminately, but commences in that part of it which immediately adjoins the scar.

The progress of recurrent Cancer after a partial removal of the breast is not exclusively organic, and does not even show a preference for that organ, but rather is centrifugal from the scar; and, when free in both directions, it tends. toward the axilla earlier than to the residue of the breast.

Cancer once established in either mamma, its primacy is thenceforth supreme. When one .breast has been .wholly or partially removed, recurrent Cancer does not spring up as 
a new disease in the opposite breast, but on the same side as the original tumour. It may, nevertheless, be possible that, a mammary Cancer having been wholly extirpated, Cancer may reappear in another part of the body, which other part may be the remaining breast. I have not met with such a case. ${ }^{1}$

It is not sufficient to remove the tumour, or any portion only of the breast in which it is situated; mammary Cancer requires the careful extirpation of the entire organ.

The situation in which the operation is most likely to be incomplete is at the edge of the mamma next the sternum.

When any texture adjoining the breast is involved in or even approached by the disease, that texture should be removed with the breast. This observation relates especially to skin, to lymphatics, to much fat, and to pectoral muscle. The attempt to save skin which is in any degree unsound is of all errors perhaps the most pernicious, and whenever its condition is doubtful, that texture should be freely removed. A broad scar, and the stretching and compression due to its subsequent contraction, appear to be especially satisfactory.

In the performance of the operation it is desirable to avoid, not only cutting into the tumour, but also seeing it. No actually morbid texture should be exposed, lest the active microscopic elements in it be set free and lodge in the wound. Diseased axillary glands should be taken away by

1 Postscript, September, 1867.-The following story illustrates the diffculty of establishing the cancerous nature of the first disease. I have lately seen a lady whose breasts have both been amputated The right mammary region is marked by two scars, and contains a soft fragment of the inner portion of the breast. At the axillary end of the scar which is in the place of the left breast, is recurrent Cancer. After the removal of the right mamma for reputed Cancer of a year's growth, four years elapsed, when there was recurrence below the niddle of the scar, but not in the remnant of the breast. The new tumour was removed, and it proved to be a mere cyst. Thenceforth the right side remained healthy. Four years later, the left breast became cancerous, and within two more years that also was removed. The wound took fifteen months in healing, and was cancerous before it closed. 
the same dissection as the breast itself, without dividing the intervening lymphatics; and the practice of first roughly excising the central mass of the breast, and afterwards removing successive portions which may be of doubtful soundness, should be abandoned. Only by deliberately reflecting the flaps from the whole mamma, and detaching it first at its edge, can the various undetected prolongations of the tumour and outlying nodules be included in the operation. To parts suspected of disease but not capable of removal it is desirable to apply the chloride of zinc. An edge of skin may be touched with the solid caustic; a paste of it may be laid on portions of the open wound ; and, however healthy in appearance, the whole remaining surface may be washed with a solution of the chloride, of a strength proportionate to the delicacy or vitality of the textures and the thickness or thinness of the flaps, and varying from twenty to forty grains in the ounce of water. By these various applications the action of the zinc may be graduated to produce the strongest caustic effect or to merely whiten the superficial textures. Regard must be had to the depth of the subjacent structures in the use of the stronger preparations, especially on the wall of the chest; and in any strength the zinc should not be in contact for more than a moment with the large veins in the axilla, lest it should soak through their thin walls, and, producing phlebitis or a chemical action on the blood, should set up a traumatic pyæmia.

The conclusions, briefly stated, are partly theoretical, and partly practical. The former are-

That the recurrence of Cancer is due to local conditions:

That these conditions are not regional, so as to belong to structures out of continuity with the first tumour:

That neither are they organic, whether as indiscriminately involving the residue of a mamma operated upon, or so as to be transferable to the second breast in consequence of the removal of that first affected :

That, on the contrary, recurrent Cancer begins near the scar:

That, when free in both directions, it tends toward the axilla earlier than to the residue of the breast : 
That, consequently, centrifugal dispersion, not organic origin, determines the recurrence of Cancer.

The practical conclusions are-

That Cancer of the breast requires the careful extirpation of the entire organ :

That the situation in which this operation is most likely to be incomplete is at the edge of the mamma next the sternum :

That, besides the breast, unsound adjoining textures, especially skin, should be removed in the same mass with the principal disease. 